\title{
Downregulation of CDCA3 expression inhibits tumor formation in pancreatic cancer
}

\author{
R. C. ZOU, Z. T. GUO, D. WEI, Z. T. SHI, Z. C. YE, G. ZHAI, C. ZHONG, B. TANG, L. WANG, J. Y. GE* \\ Department of Hepatobiliary Surgery, The Second Affiliated Hospital of Kunming Medical University, Kunming, Yunnan, China \\ ${ }^{*}$ Correspondence: gejiayunfey@126.com
}

Received April 11, 2020 / Accepted April 29, 2020

\begin{abstract}
Downregulation of cell division cycle-associated 3 (CDCA3) markedly inhibited cell growth and induced apoptosis in tumors. However, the effect of CDCA3 in pancreatic cancer (PAC) was rarely investigated. Therefore, this study attempted to clarify the role of CDCA3 in PAC. The mRNA and protein expression of CDCA3 were examined in PAC cell lines and tumor tissues by using real-time quantitative PCR (RT-qPCR), western blotting (WB), and immunohistochemistry (IHC). The effects of CDCA3 downregulation on cell proliferation, apoptosis, and colony information were investigated through MTT assay, Annexin V-APC single staining cell apoptosis detection, and colony formation test. The microarray and ingenuity pathway analysis were employed to explore the potential regulatory relation. The tumor xenograft model was established for determining the effect of CDCA3 downregulation on the growth of PAC in vivo. The results showed that the expression of CDCA3 in tumor tissues was higher than that of normal tissues $(\mathrm{p}<0.05)$. In addition, the mRNA expression of CDCA3 was markedly increased in PANC-1 cells and SW 1990 cells when compared with human pancreatic duct epithelial (HPDE) cells $(\mathrm{p}<0.05)$. MTT assay showed that the cell proliferation of PANC-1 cells and SW 1990 cells was significantly inhibited after the lentivirus transfection of CDCA3 knockdown $(\mathrm{p}<0.05)$. Annexin V-APC apoptosis assays suggested that the apoptotic cell number was markedly increased in the shCDCA3 group compared to that in the shCtrl group in SW 1990 cells and PANC-1 cells $(\mathrm{p}<0.05)$. Meanwhile, the activity of caspase-3/7 was obviously elevated in the shCDCA3 group compared to the shCtrl group $(\mathrm{p}<0.05)$. The colony formation was notably inhibited in the shCDCA3 group relative to the shCtrl group in SW 1990 cells $(\mathrm{p}<0.05)$. Moreover, the tumor growth was evidently suppressed in the shCDCA3 group compared with the shCtrl group in vivo $(\mathrm{p}<0.05)$. These findings revealed that CDCA3 plays a crucial role in the progress of PCA by regulating cell apoptosis and proliferation, which may serve as a potential target for PAC treatment.
\end{abstract}

Key words: cell division cycle-associated 3, pancreatic neoplasms, cell proliferation, apoptosis

Pancreatic cancer (PAC) is a fatal malignant tumor gravely threatening human health worldwide $[1,2]$. World widely speaking, PAC is the 11th most common cancer including 458,918 new cases and causing 432,242 deaths based on GLOBOCAN 2018 estimates [3]. Currently, the incidence and mortality of PAC are estimated to increase globally [4]. Moreover, according to the latest statistics released by the American Cancer Society, the United States had witnessed approximately 55,440 newly diagnosed cases, as well as 44,330 deaths in 2018 [5]; and PAC mortality and morbidity raised unceasingly in China in recent years $[6,7]$. Etiologic factors of PAC mainly comprise of smoking, alcoholism, heredity, diabetes, and obesity $[5,8]$. The major symptoms of PAC include unexplained upper abdominal pain, icterus, pruritus, anorexia, and weight loss [9]. These atypical symptoms may directly contribute to the misdiagnosis at the early stage of the disease. Generally, computed tomography
(CT), magnetic resonance imaging (MRI), and ultrasound have been frequently used to detect PAC [10], and therapeutic paradigms of PAC consist of surgery, radiation therapy (RT), and chemotherapy [11]. However, the prognosis of PAC is still worst among all cancers and the 5-year survival rates are usually less than 5\% derived from its late diagnosis, rapid progression, early metastasis, and resistance to available therapeutic strategies $[12,13]$. Currently, the mounting evidence revealed the aberrant versions of cytokines implicated in the dysfunction of the cell cycle. A previous study suggested that the downregulation of taurine upregulated 1 (TUG1) blocked the cell cycle and suppressed the proliferation of PAC cells [14]. Similarly, cyclin D1 induced the progression of G1/S transition and cell proliferation in PAC by mediating the activity of cyclin D1-CDK4 complexes and retinoblastoma (Rb) expression [15]. Moreover, cell division cycle-associated 3 (CDCA3) could regulate mitotic 
entry [16] and effectively destroy wee1, the mitosis-inhibiting kinase that served as one part of the S-phase kinaseassociated protein 1/Cullin 1/F-box (SCF) E3 ubiquitin ligase complex, and then impart an important effect on the cell cycle progression $[17,18]$. Numerous studies documented that the expression of CDCA3 was obviously upregulated in tumors including liver cancer [19], prostate cancer [20], and oral squamous cell carcinoma [21]. Adams et al. found that depletion of CDCA3 expression markedly suppressed the development of non-small cell lung cancer (NSCLC) and induced its senescence [22]. Meanwhile, some research showed that overexpression of $\mathrm{CDCA} 3$ was closely related to oral squamous cell carcinoma (OSCC) progression via avoiding the cell cycle arrest at the G1 phase and reducing the expression of cyclin-dependent kinase inhibitors [21]. In the current work, our findings indicated that the CDCA3 expression was significantly elevated in PAC, and CDCA3 expression downregulation significantly inhibited tumorigenesis and cell proliferation. Additionally, the lower expression of CDCA3 correlated with higher survival rates. Therefore, these results indicated that CDCA3 might serve as a new candidate target and diagnostic criteria for PAC treatment.

\section{Materials and methods}

Cell cultures. The human pancreatic cancer cell lines, including normal pancreatic duct epithelial (HPDE) cells, SW 1990, PANC-1, and AsPC-1 cells were purchased from the Cell Bank (The Chinese Academy of Sciences, Shanghai, China). These cells had then been maintained in the RPMI-1640 medium containing $10 \%$ fetal bovine serum (FBS), $100 \mathrm{IU} /$ $\mathrm{ml}$ penicillin as well as $100 \mu \mathrm{g} / \mathrm{ml}$ streptomycin.

Construction of CDCA3 knockdown lentivirus. shCDCA3 and control shRNA (shCtrl) were designed by Shanghai Jikai Genetic Chemistry Technology Co., Ltd. (Shanghai, China). The following sequences were used in this study: 5'-TATCTGTTGAGGAACAGAT-3'; 5'-TTCTCCGAACGTGTCACGT-3'. The shRNAs $(100 \mathrm{ng} / \mu \mathrm{l})$ were separately inserted into the plasmid GV115 (Shanghai Jikai Gene Technology Co., LTD, Shanghai, China). Recombinant lentiviruses were generated through the transfection of $293 \mathrm{~T}$ cells with $20 \mu \mathrm{g}$ GV115 plasmid and the helper plasmids (Shanghai Jikai Gene Technology Co., LTD, Shanghai, China) in a $10 \mathrm{~cm}$ dish at $80 \%$ confluency by using Lipofectamine ${ }^{\circ}$ 2000. Thereafter, viral supernatant was collected and centrifuged at $4^{\circ} \mathrm{C}$ and $10,000 \mathrm{rpm}$ for removing the cell debris, then filtered by using polyvinylidene fluoride membranes after transfecting for $48 \mathrm{~h}$. After $72 \mathrm{~h}$ of transfection, green fluorescent protein (GFP) expression was observed, and the CDCA3 expression was detected through western blotting.

Real-time quantitative PCR (RT-qPCR). Total RNA was collected by the total RNA Kit (Invitrogen; Thermo Fisher Scientific, Inc., Waltham, MA, USA) and transcribed into cDNA with a cDNA Synthesis Kit (Promega, Madison, WI) in accordance with the manufacturer's instructions. Table 1 shows the primer sequences used in this study. SYBR Green assay (Roche Ltd., Basel, Switzerland) was used to carry out RT-qPCR with the Rotor-Gene 3000 system (Corbett Research, Australia). The RT-qPCR amplification conditions were showed below: initial denaturation for $30 \mathrm{~s}$ at $95^{\circ} \mathrm{C}$, followed by 40 cycles of denaturation for $5 \mathrm{~s}$ at $95^{\circ} \mathrm{C}$, and then 40 cycles of amplification for $30 \mathrm{~s}$ at $60^{\circ} \mathrm{C}$. The $2^{-\Delta \Delta \mathrm{Ct}}$ method was employed to analyze data, and GAPDH was used as an internal reference.

Western blotting. Cells were lysed for 10 minutes using the RIPA buffer $(1 \mathrm{ml}$, Thermo Fisher Scientific, Waltham, MA, USA), and centrifuged to collect the supernatant. Total protein was calculated through the BCA Protein Assay Kit (Shanghai Biyuntian Biotechnology Co., Ltd., Shanghai, China). Proteins were subjected to electrophoresis, followed by the transfer onto the polyvinylidene fluoride (PVDF) membrane (Millipore, Billerica, MA, USA). Then, the membrane was blocked with 5\% skim milk powder for $1 \mathrm{~h}$ under ambient temperature, followed by $18 \mathrm{~h}$ of incubation at $4^{\circ} \mathrm{C}$ using the primary antibodies displayed in Table 2. GAPDH served as the internal reference. Subsequently, all membranes were rinsed in TBST for an incubation using the secondary antibody. The chemiluminescent signal was detected using Alpha Innotech (Bio-Rad Laboratories, Berkeley, CA, USA) with the ECL method.

Patients and tissue samples. 10 samples of tumor tissues and 8 samples of adjacent tissues were acquired from patients in tumor resection surgery of the Second Affiliated Hospital of Kunming Medical University. Based on the American Joint Committee on Cancer, all patients were histologically diagnosed as PAC. Each of the patients has written consent. This study was approved by the Ethics Committee at the Second Affiliated Hospital of Kunming Medical University.

Immunohistochemical staining. Tissues were cut to 5 $\mu \mathrm{m}$ sections after formalin fixation and paraffin embedding. Immunohistochemical (IHC) staining was carried out to investigate the histomorphological features and that procedure was referred to as diaminobenzidine staining using the Dako EnVision detection system (Dako, Denmark). The heating sodium citrate buffer $(10 \mathrm{mM}, \mathrm{pH}$ 6.0) was used for antigen retrieval for $30 \mathrm{~min}$. And a primary antibody of CDCA3 (1:100, Sigma, SAB4503278) was diluted by $0.05 \mathrm{M}$ Tris-buffered saline. Then, the negative control was achieved by not using the primary antibody. A DM2500 (Leica) was used to photograph the slides. According to the previous report [23], QuantCenter 2.0 (3DHISTECH Ltd., Hungary) was used to calculate the histochemistry score (H-Score), which included the intensity of staining $(0=$ negative; $1=$ weak; 2 = moderate; 3 = strong).

Celigo ${ }^{\circ}$ cell counts. Cells were seeded into the 96-well plate at $2 \times 10^{3}$ cells/well, and transfected with either green fluorescence labeled non-targeting control siRNA $(50 \mathrm{nM})$, or targeting siRNA (siCDCA3, $50 \mathrm{nM}$ ) using Lipofectamine 2000 (Thermo Fisher Scientific). After culturing for 1 d, 2 d, $3 \mathrm{~d}, 4 \mathrm{~d}$, and $5 \mathrm{~d}$, a cytometer Celigo ${ }^{\circ}$ was used to count cells. 
Table 1. Information of primer sequences.

\begin{tabular}{|c|c|c|c|}
\hline Gene & Forward primer & Reverse primer & Length (bp) \\
\hline CDCA3 & 5'-ATTGCACGGACACCTATGA-3' & 5'-TGTGGGCTGTCTTGCTTC-3' & 261 \\
\hline BIK & 5'-GGAGGACTTCGATTCTTTGG-3' & 5'-GAAACCGTCCATGAAACTTCTA-3' & 208 \\
\hline CAV1 & 5'-CTGAGCGAGAAGCAAGTG-3`3 & 5'-AGAGAGAATGGCGAAGTAAATG-3' & 264 \\
\hline CLIC4 & 5'-CTGGATGGCAATGAAATGA-3’ & 5’-TGGTGAACTCGTCCCTACT-3‘ & 160 \\
\hline CALU & 5'-GTTTGTTGAGTTTCGGGATA-3' & 5'ATCATAGTCTGAGGGAAGGA-3' & 85 \\
\hline FRS2 & 5'-TCAGACGCCCAAGTTTAGAA-3' & 5'-TGGTAGGGGTTTGTGGAAGG-3‘ & 126 \\
\hline HIPK2 & 5'-TGACAGCGTGTTCTTAGGAC-3‘ & 5'AGGAAAGTGGGAGTATGATTAGG-3‘ & 91 \\
\hline $\mathrm{CDC} 42$ & 5'-CTTTCTTGCTTGTTGGGACT-3' & 5'-TAGGCTTCTGTTTGTTCTTGG-3` & 84 \\
\hline CITED2 & 5'-GACAAACCAGCACTTCCG-3' & 5'-CСАAАCССАТTTCTATCAC-3' & 269 \\
\hline CYP1A1 & 5'-CACССTCATCAGTAATGGTCAGA-3' & 5'-AACGTGCTTATCAGGACCTCA-3‘ & 186 \\
\hline TGFBR1 & 5’-ACGGCGTTACAGTGTTTCTG-3` & 5'-GCACATACAAACGGCCTATCTC-3‘ & 167 \\
\hline MAPK9 & 5'AGCCAACTGTGAGGAATTATGT-3' & 5'-GCTTGTCAGGATCAATCACTAAC-3‘ & 98 \\
\hline HMMR & 5'ACAAGTGGCGTCTCCTCTATG-3‘ & 5'-AGCTGCACCATGTTCATTCA-3‘ & 113 \\
\hline TIMP3 & 5'TGGGTTGTAACTGCAAGATCA-3‘ & 5'-GGTAGCCAGGGTAACCGAAA-3، & 111 \\
\hline SERPINE2 & 5'AACGCCGTGTTTGTTAAGAATG-3‘ & 5'-CGTGATTTCCACAGACCCTTG-3' & 254 \\
\hline CDC25A & 5’-TTGGTGGATTTTGAAGGT-3‘ & 5'-AGTGAAGCCGTGATGGTA-3‘ & 233 \\
\hline PIK3R1 & 5'-ACCACTACCGGAATGAATCTCT-3‘ & 5'-GGGATGTGCGGGTATATTCTTC-3‘ & 207 \\
\hline SMC1A & 5'-AGCGAAAGGCAGAGATAATGG-3' & 5'-GGTAGTCAAGAGGCAAGAAGG-3' & 234 \\
\hline INHBB & 5'-CACCGGCTACTACGGGAACT-3' & 5'-TGTGGAAGGAGGAGGCAGAG-3' & 83 \\
\hline PTGS2 & 5’-CTCCTGTGCCTGATGATTGC-3‘ & 5'-CAGCCCGTTGGTGAAAGC-3' & 215 \\
\hline ACVR1 & 5'-CCGTTCTACGATGTGGTTCC-3' & 5'-GAGGTTAATGTCGGGTCTGA-3' & 119 \\
\hline TP53INP1 & 5'--3’CACTCСТCАССТCGCATC & 5'--3’AGCCAACCTGTCACTCTTC & 220 \\
\hline BMPR1B & 5'--3’TGACATACCACCTAACACTC & 5'--3’ATTCTTCCACTATACСТCСТ & 176 \\
\hline PPM1D & 5'--3'CTACACCACCAGTCAAGTCAC & 5'--3’AGAAGGCATTGCTACGAACC & 93 \\
\hline SESN2 & 5'--3’TCTTACCTGGTAGGCTCCCAC & 5'--3’AGCAACTTGTTGATCTCGCTG & 122 \\
\hline THBS1 & 5'--3’AGACTCCGCATCGCAAAGG & 5'--3’TCACCACGTTGTTGTCAAGGG & 157 \\
\hline CEBPD & 5'--3’AGAGCGCCATCGACTTCAG & 5'--3’CGCCTTGTGATTGCTGTTG & 110 \\
\hline SGK1 & 5'--3'GAAGCAGAGGAGGATGGGTCT & 5'--3'GTTTAGCATGAGGATTGGACG & 200 \\
\hline ACVR2B & 5’--3'AGACACGGGAGTGCATCTACT & 5'--3'GCCTATCGTAGCAGTTGAAGTC & 189 \\
\hline RCHY1 & 5'--3'TAGAGGCTACAGATGTCCATTA & 5'--3'GCAGAGAATATCCACAGTCAT & 133 \\
\hline MKI67 & 5'--3'GGAACAGCCTCAACCATCAG & 5'--3'ССАСТСТТТСТСССТССТСТС & 210 \\
\hline GAPDH & 5'--3'TGACTTCAACAGCGACACCCA & 5'--3'CACCCTGTTGCTGTAGCCAAA & 121 \\
\hline
\end{tabular}

Table 2. Antibody list.

\begin{tabular}{|c|c|c|c|c|c|}
\hline Antibodies & Manufacturer & Source & Reactivity & Dilution & Catalog numbers \\
\hline CDCA3 & Abcam & Rabbit & Human & $1: 500$ & ab167037 \\
\hline SMC1A & Abcam & Rabbit & Human & $1: 1000$ & ab133643 \\
\hline PTGS2 & Abcam & Rabbit & Human & $1: 500$ & ab15191 \\
\hline MKI67 & Abcam & Rabbit & Human & $1: 1000$ & ab15580 \\
\hline SESN2 & Abcam & Rabbit & Human & $1: 200$ & ab57810 \\
\hline p53 & CST & Rabbit & Human & $1: 1000$ & \#2527 \\
\hline p-p53 & Abcam & Rabbit & Human & $1: 1000$ & \#9284 \\
\hline GAPDH & Santa-Cruz & Mouse & Human & $1: 1000$ & sc- 32233 \\
\hline Anti-Rabbit IgG & Santa Cruz & goat & Rabbit & $1: 2000$ & sc-2004 \\
\hline Anti-Mouse IgG & Santa-Cruz & Goat & Mouse & $1: 2000$ & sc-2005 \\
\hline
\end{tabular}

MTT assay. PANC-1 cells and SW 1990 cells were seeded into 96 well plates with $2 \times 10^{3} /$ well. SW 1990 cells and PANC-1 cells were treated with $0.2 \mathrm{mg} / \mathrm{ml}$ of 3-(4,5-dimethyl-2-thiazolyl)-2,5-diphenyl-2-H-tetrazolium bromide (MTT, Sigma) for $4 \mathrm{~h}$ following transfection.
Then, cells were centrifuged (1000 rpm, $10 \mathrm{~min}$ ), and the supernatant was discarded. Dimethyl sulfoxide (DMSO, Sigma, $200 \mu \mathrm{l} /$ well) was used for dissolving crystal, and then the optical density of the solution was determined at the wavelength of $570 \mathrm{~nm}$. 
Annexin V-APC single staining to detect cell apoptosis. SW 1990 cells and PANC-1 cells were rinsed once again by using PBS following centrifugation (1300 rpm, $3 \mathrm{~min}$ ). Annexin V-APC $(10 \mu \mathrm{l}$, eBioscience) was added into $1 \times$ binding buffer $(200 \mu \mathrm{l})$ to prepare the mixture, then cells were resuspended within the mixture for 15 min under ambient temperature. Before flow cytometry analysis, $400 \mu \mathrm{l}$ $1 \times$ binding buffer was added.

Caspase activity determination. The Caspase Assay System was used for testing the activity of DEVDase (caspase-3/7). SW 1990 cells and PANC-1 cells were seeded into 96 well plates with $1 \times 10^{4}$ per well. After transfection, PANC-1 cells were treated with $100 \mu$ l Caspase-Glo from the kit and shocked at a speed of $300 \mathrm{rpm}$ for $30 \mathrm{~min}$, and then incubated at room temperature for $3 \mathrm{~h}$. The reaction product was examined by the Athos 2001 automated plate reader (Athos Labtec Instruments, UK) at a wavelength of $405 \mathrm{~nm}$.

Colony formation. Cell viability was detected by colonyforming assay. After $24 \mathrm{~h}$ transfection, an ImageJ (V1.51t, National Institutes of Health, Bethesda, MD, USA) was utilized for counting digested cells. Simply, $1 \times 10^{3}$ cells were seeded into the 6-well plates to culture for a period of 10-14 days $\left(37^{\circ} \mathrm{C}, 5 \% \mathrm{CO}_{2}\right)$. And crystal violet was used to stain the colonies, and then colonies were counted. All experiments were repeated three times.

Microarray and ingenuity pathway analysis. Total RNA sample quality was estimated using the Agilent Bioanalyzer 2100 (Agilent, Santa Clara, CA, USA), and then the NanoDrop 2000 (Thermo Fisher Scientific, Waltham, MA, USA) was used to quantify the purified RNA. In addition, microarray data were processed by the human GeneChip prime view (Affymetrix, Santa Clara, CA, USA), and gene expression determining was complied with the manufacturer's instructions. R software platform (v.2.13.0) was used to analyze microarray data, and the limma package was applied in $\mathrm{R}$ to correct microarray data background and normalize quantile $[24,25]$. A p-value $<0.05$ and $(|\mathrm{FC}|)>$ 1.5 were chosen as principles, then significant differentially expressed genes (DEG) between shCDCA3 and shCtrl group of SW 1990 cells were screened out. DEG was demonstrated by hierarchical cluster analysis and heatmap. And DEG was categorized in specific diseases and functions, canonical pathways, and gene networks by IPA [26]. When the pathway is significantly activated, the positive Z-score was set as a representative, whereas negative Z-score was considered as significantly suppressed, and a significant index was $\mid$ Z-score $\mid>2$ [27].

Xenograft experiment. For xenografts, female BALB/c nude mice (4-weeks old) were provided by Shanghai Lingang Biotechnology Co., Ltd. The Animal Care and Use Committee of Shanghai Jikai Genetic Chemistry Technology Co., Ltd. (Shanghai, China) had approved all experimental protocols. Each animal was kept within the specified pathogen-free facility and raised according to the guidelines for laboratory animal care. SW 1990 cells were subjected to
$24 \mathrm{~h}$ of shCDCA3 (50 nM) or shCtrl transfection; then, 100 $\mu \mathrm{l}$ PBS was used to suspend cells to the concentration of $4 \times$ $10^{6} \mathrm{cells} / \mathrm{ml}$, and the cells were injected into the right flank of female athymic BALB/C nude mouse ( $\mathrm{n}=6$, each group). When inoculation of the pancreatic cancer cells for 28 days, intraperitoneal injection of $10 \mu \mathrm{l} / \mathrm{g}$ D-Luciferin (Shanghai qcbio Science and Technologies co., Ltd., Shanghai, China) to the mice was carried out, and mice were anesthetized with $70 \mathrm{mg} / \mathrm{kg}$ pentobarbital sodium by intraperitoneal injection after $15 \mathrm{~min}$. Then, the anesthetized mice were transferred to the small animal live-imaging system (LT Lumina; PerkinElmer, Inc., Waltham, MA, USA) for testing the intensity of fluorescence. Finally, all animals were sacrificed, and the tumor width (W), as well as length (L), was measured using the calipers, and the tumor volume was calculated by the following formula: $\left(\mathrm{L} \times \mathrm{W}^{2}\right) \times 0.5$.

Statistical methods. SPSS 19.0 (IBM Corporation, NY, USA) was used for data analysis. Data were expressed in the form of the mean \pm standard deviation. Boxplots as well as Cox regression analysis was used for comparing data on patient survival that were collected based on the GEPIA database. ANOVA, together with Tukey's post hoc multiplecomparison was employed for comparisons among multiple groups. A p-value $<0.05$ indicated statistical significance.

\section{Results}

Upregulation of CDCA3 relates to a dismal prognosis in PAC patients. GEPIA database (http://gepia.cancer-pku. cn) including 179 pancreatic tumor tissue and 171 normal tissues were used to analyze the CDCA 3 expression. The results showed that the CDCA3 level of tumor tissue was higher than that of normal tissue (Figure $1 \mathrm{~A}, \mathrm{p}<0.05$ ). The mRNA expression of CDCA3 in PANC-1, SW 1990, and AsPC-1 cells was significantly increased compared to HPDE cells (Figure 1B, $\mathrm{p}<0.05$ ). Meanwhile, western blotting and RT-qPCR were carried out to determine the CDCA3 level following the lentivirus transfection. The results indicated that the mRNA and protein expression of CDCA3 were markedly downregulated in the shCDCA3 group than the shCtrl group (Figures $1 \mathrm{C}-1 \mathrm{~F}, \mathrm{p}<0.05$ ). Then, IHC was employed to estimate CDCA3 expression in tumor tissue and normal tissue. The graphs showed that the CDCA3 stained strongly positive in tumor tissue when compared to that of in normal tissue (Figures 1G, 1H; $\mathrm{p}<0.05$ ). Serial analyses (Kaplan-Meier) indicated that the overall survival of the Low CDCA3 group was overtly longer than the High CDCA3 group (Figure 1I; $\mathrm{p}<0.05$ ).

Downregulation of CDCA3 significantly inhibited cell proliferation and vitality. Celigo Image Cytometer and MTT assay were employed to detect the effect of CDCA3 on the proliferation and vitality of PAC cells. PAC cell viability was measured by Celigo Image Cytometer on $1 \mathrm{~d}$, $2 \mathrm{~d}, 3 \mathrm{~d}, 4 \mathrm{~d}$, and $5 \mathrm{~d}$ after transfection. The cell proliferation of the shCDCA3 group was remarkably reduced than 
A

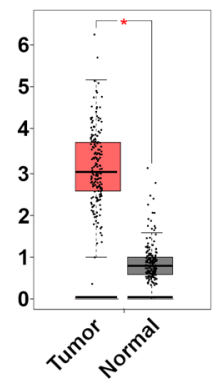

$\mathbf{F}$

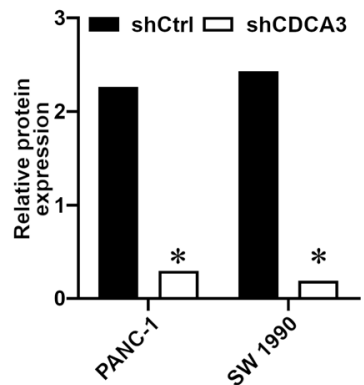

B

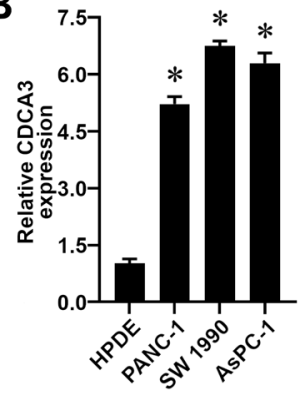

G

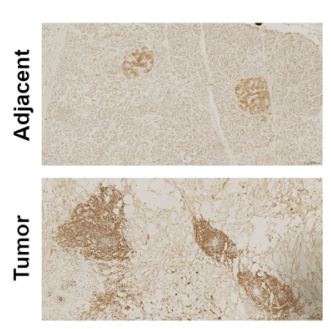

C

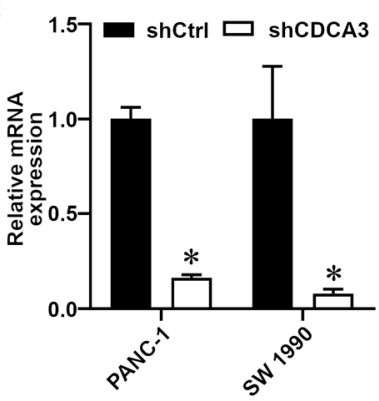

D

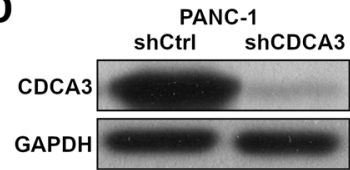

$\mathbf{E}$

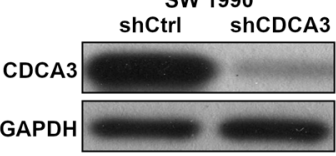

H

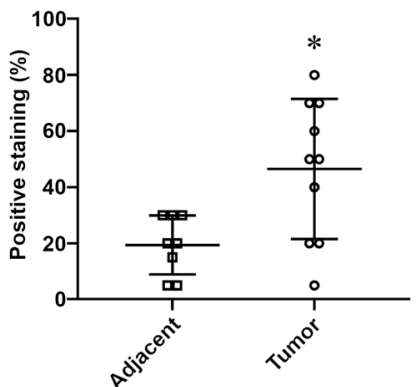

1

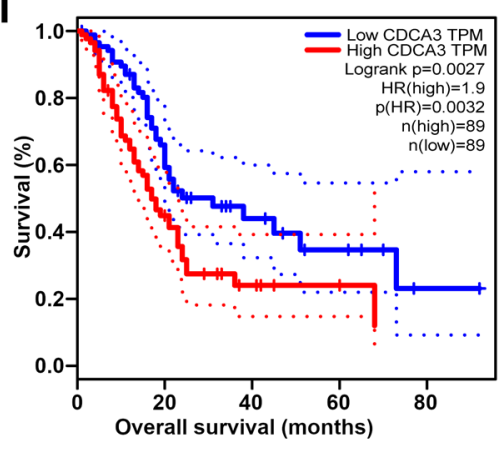

Figure 1. The expression of CDCA3 in patients with PAC. A) The CDCA3 expression in normal and PAC tissues; B) The mRNA expression of CDCA3 in different cell lines including HPDE, SW 1990, PANC-1, and AsPC-1 cells; C-F) The mRNA and protein expression of CDCA3 in SW 1990 and PANC-1 cells after lentiviral transfection; G) Representative IHC staining images for CDCA3 (Magnification $\times 200$ ), $100 \mu \mathrm{m}$ bar is shown as a scale; $\mathrm{H}$ ) Quantification of CDCA3 staining score; I) The survival curves of PAC patients obtained from GEPIA analysis. ${ }^{*} \mathrm{p}<0.05$, indicated statistically significant difference.
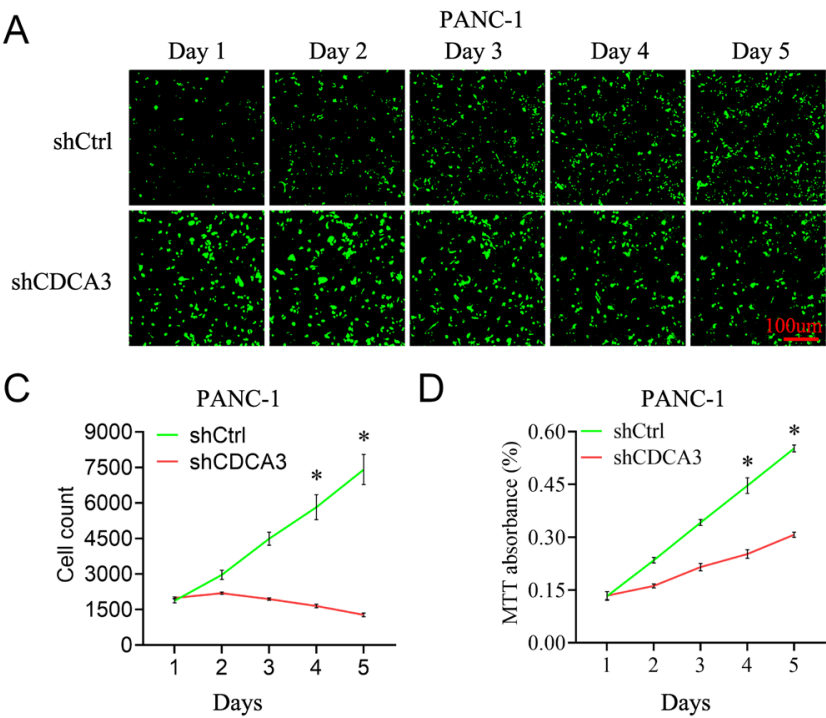

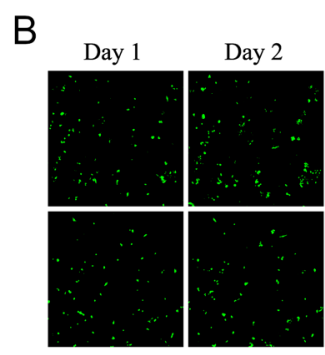

E
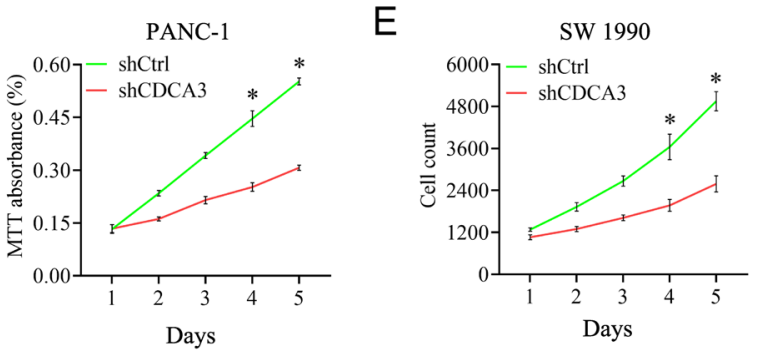

SW 1990

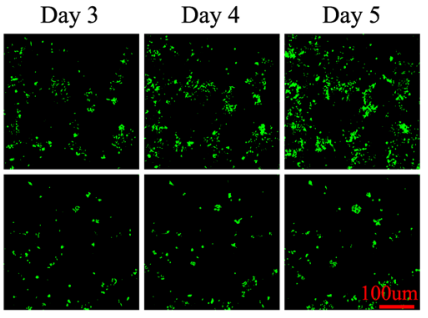

F

$\mathrm{F}$ SW 1990

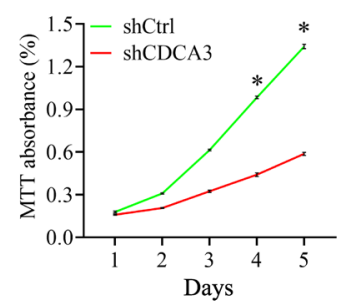

Figure 2. Effect of CDCA3 downregulation on the proliferation and activity of PAC cells in vitro. A, B) Typical Immunofluorescence image for CDCA3 in PANC-1 and SW 1990 cells; C, D) Cell proliferation and activity of PAC cells of PANC-1 cells; E, F) Cell proliferation and activity of SW 1990 cells. ${ }^{*} \mathrm{p}<0.05$, compared with shCtrl group.

the shCtrl group in PANC-1 and SW1990 cells, respectively (Figures 2A, 2B). The numbers of cells were obviously downregulated in the shCDCA3 group compared with the shCtrl group at $4 \mathrm{~d}$ and $5 \mathrm{~d}$ (Figures $2 \mathrm{C}, 2 \mathrm{E} ; \mathrm{p}<0.05$ ).
Additionally, MTT assay showed that cell vitality of SW 1990 cells and PANC-1 cells was significantly inhibited in the shCDCA3 group compared to the shCtrl group at $4 \mathrm{~d}$ and $5 \mathrm{~d}$ (Figures 2D, 2F; p $<0.05$ ). 
Downregulation of CDCA3 promoted cell apoptosis and inhibited the colony formation. Annexin V-APC apoptosis and caspase $3 / 7$ activity assays were carried out for detecting the apoptosis of PAC cells following lentivirus transfection. The results indicated that the apoptotic cell number was markedly increased in the shCDCA3 group than the shCtrl group in PANC-1 and SW 1990 cells (Figures $3 \mathrm{~A}-3 \mathrm{C}, \mathrm{p}<0.05)$. Meanwhile, the caspase-3/7 activity was obviously elevated in the shCDCA3 group compared to the shCtrl group in PANC-1 and SW 1990 cells (Figure 3D, $\mathrm{p}<0.05)$. Similarly, colony formation was notably inhibited in the shCDCA3 group compared with the shCtrl group in PANC-1 and SW 1990 cells (Figures 3E, 3F; p<0.05).

Downregulation of CDCA3 associated with multiple pathways changed in SW 1990 cells. RNA-Seq analysis results showed that 1862 genes had significant differential expression including 370 upregulated genes and 586 downregulated genes in SW 1990 cells after transfecting with shCDCA3 (Figure 4A). Meanwhile, ingenuity analysis of pathways was carried out, and the results showed that differently expressed genes mainly enriched in 12 signal transduction pathways (Figure 4B). Xbp1 markedly changed after the CDCA3 knockdown accompanied by 22 coherently suppressed genes (Figure 4C), and KLF6 inhibited the movement and migration of tumor cell line through CYP1A1, PLAU, PTGS2, and TGFBR1 (Figure 4D). Analysis of the functional interaction network was carried out and the relationship among genes belonging to certain diseases or functions is shown in Figure 4E. The expression of thirty differentially expressed genes was tested by using RT-qPCR assay. The results showed that the mRNA level of PTGS2, MKI67, SMC1A, HMMR, TIMP3, CLIC4, and CALU was downregulated (Figures 4A, $4 \mathrm{~F})$. Moreover, the protein expression of five differentially expressed genes was investigated through western blotting. And the results suggested that SENS2, SMC1A, and PTGS2 expression was downregulated (Figures 4G, 4H).

CDCA3 silencing suppressed the in vivo tumor growth. To investigate the relationship between CDCA3 expression and tumor growth, a tumor xenografts model was established, and RT-qPCR was used to verify the CDCA3 level before establishing the model. The results showed that the mRNA expression of CDCA3 was markedly downregulated in the shCDCA3 group than in the shCtrl group (Figure 5C; $\mathrm{p}<0.05$ ). And the fluorescence signals of tumor xenografts were observed and the total radiation efficiency was calculated by the recorded fluorescence signal in the shCDCA3 and the shCtrl groups, respectively. Fluorescence imaging results suggested that total radiation efficiency in the shCDCA3 group was apparently decreased compared with that of in the shCtrl group (Figures 5A, 5D; $\mathrm{p}<0.05$ ).
A
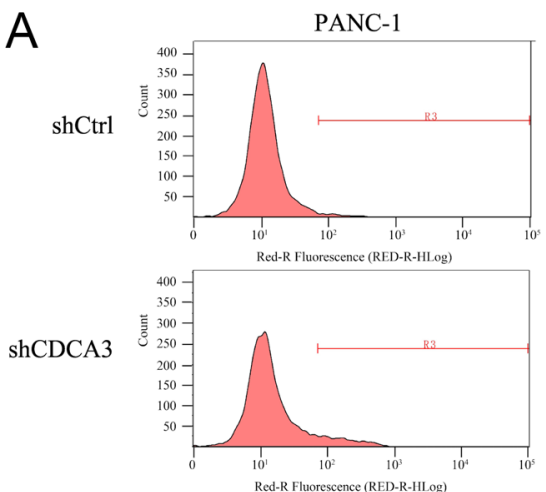

D

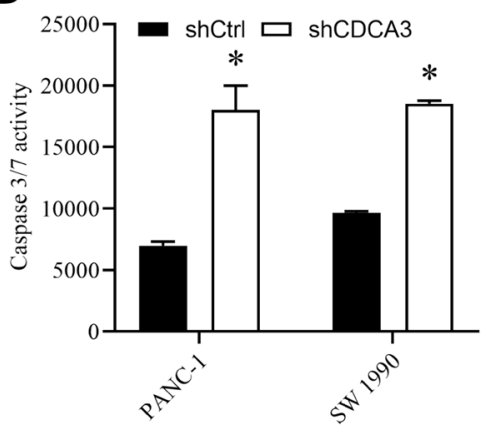

B
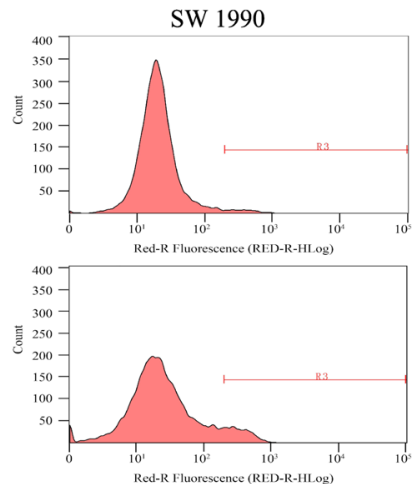

$E$

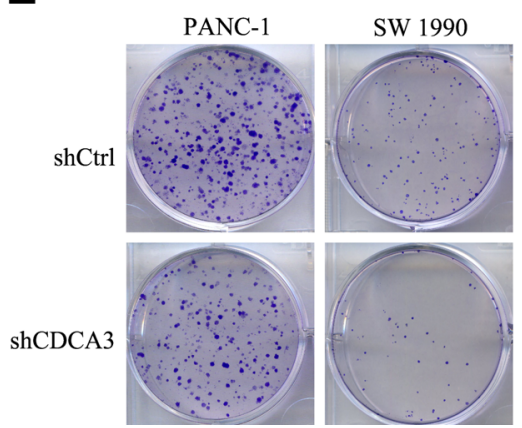

C

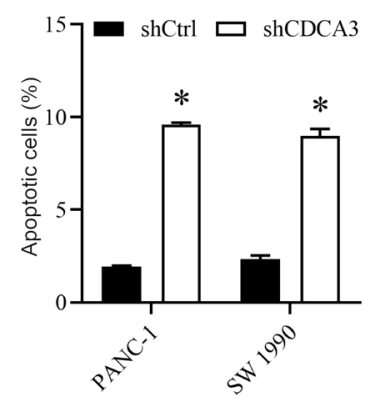

$\mathrm{F}$

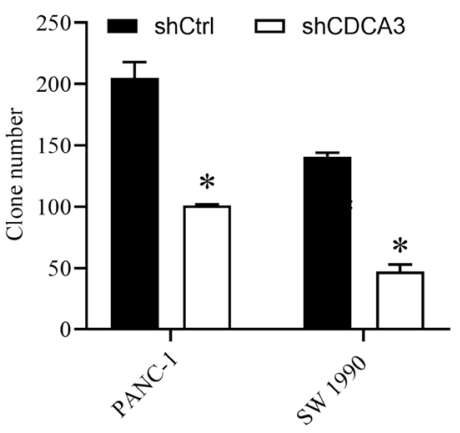

Figure 3. Effects of CDCA3 downregulation on apoptosis and colony formation in vitro. A-C) Flow cytometry assay for detecting apoptotic cells; D) Caspase activity assay to evaluate apoptosis; E, F) Representative colony formation images in PANC-1 and SW1990 cells. ${ }^{\star}$ p $<0.05$, compared with shCtrl group. 


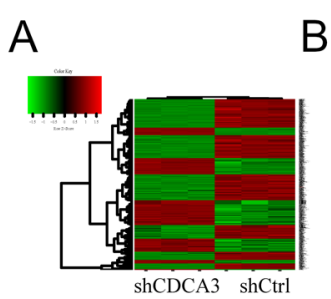

B
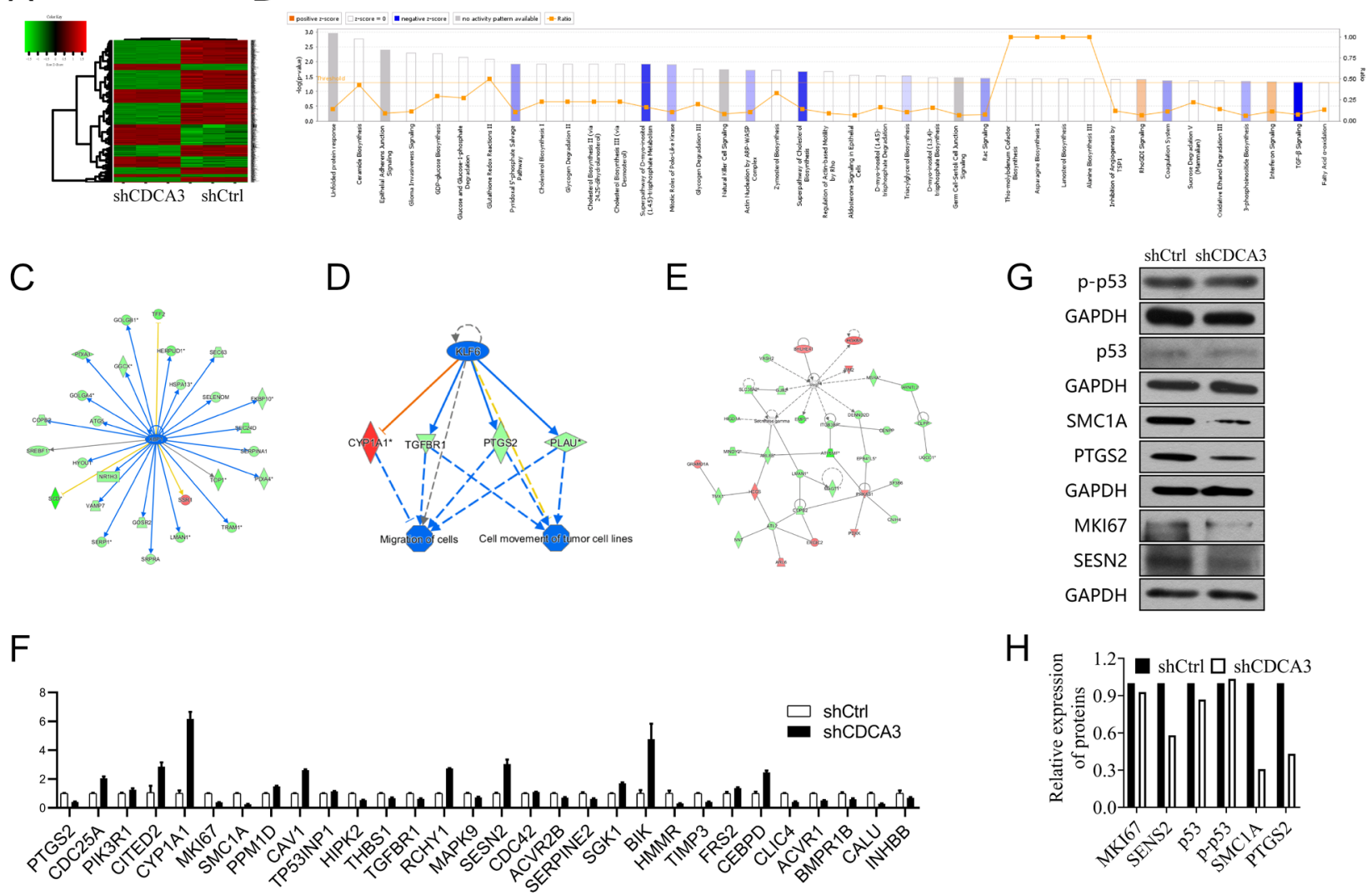

Figure 4. CDCA3 downregulation related to a variety of genes expression changes. A) Heat map analysis of differentially expressed genes between the shCDCA3 group and the shCtrl group; the color scale represents the relative expression of genes in certain slide: green indicates low relative expression levels; red indicates high relative expression levels; black indicates zero (no change); B) Histogram of the signaling pathway was drawn based on IPA pathway, and gene differential level was represented as a ratio, which was the gene ratio in this signal pathway members; $\mathrm{C}$, $\mathrm{D}$ ) The role of $\mathrm{Xbp} 1$ and KLF6 predicted by bioinformatic methods; E) A network of interactions between molecules related to certain diseases or functions. Downregulated and upregulated genes were represented by green and red color, respectively. Confirmed regulatory relationship was represented by solid arrow, and predicted regulatory relationship was represented by dotted line. ' $T$ ' bar was used to represent inhibitory relationship; F) RT-qPCR analysis for validating the (DEG) of the Array data; $(G, H)$ Western blotting analysis for the (DEG).

The tumor volume and tumor growth rate were markedly decreased in the shCDCA3 group when compared to the shCtrl group at 18 days after cell injection (Figures 5B, 5E; $\mathrm{p}<0.05$ ). And the tumor weight was evidently decreased in the shCDCA3 group relative to the shCtrl group (Figures 5B, 5F; $\mathrm{p}<0.05)$.

\section{Discussion}

Pancreatic cancer (PAC) is a devastating metastatic disease that severely threatens human health [28]. The epidemiological investigation identified that the incidence and mortality of PAC raised year by year all over the world $[29,30]$. Currently, researches showed that smoking, obesity, diabetes mellitus, gene susceptibility, and occupational exposures were the major factors contributing to PAC [31]. Even though substantial continuing progress has been achieved, it remains a major reason for the cancer- associated death worldwide derived from insufficiency of effective therapy methods against its high malignancy and metastatic ability [32]. Therefore, seeking effective treatment strategies was conducted to alleviate patient's symptoms and elevate survival rates in patients with PAC. According to our results, CDCA3 expression within PAC tissue was obviously upregulated compared with that in normal ones. Besides, the similar results were obtained from PAC cell lines including SW 1990 and PANC-1 PAC cells. Survival analysis indicated a distinct inverse relationship of CDCA3 expression with overall survival. Meanwhile, the downregulation of CDCA3 inhibited the cell proliferation and promoted the apoptosis indicated by Annexin V-APC single staining and Celigo ${ }^{\circ}$ cell counts assay. Moreover, according to the xenograft tumor growth experiments, tumor growth was remarkably suppressed in the shCDCA3 group relative to the shCtrl group. All the above findings indicated that CDCA3 played an important part in oncogenesis as well as the devel- 


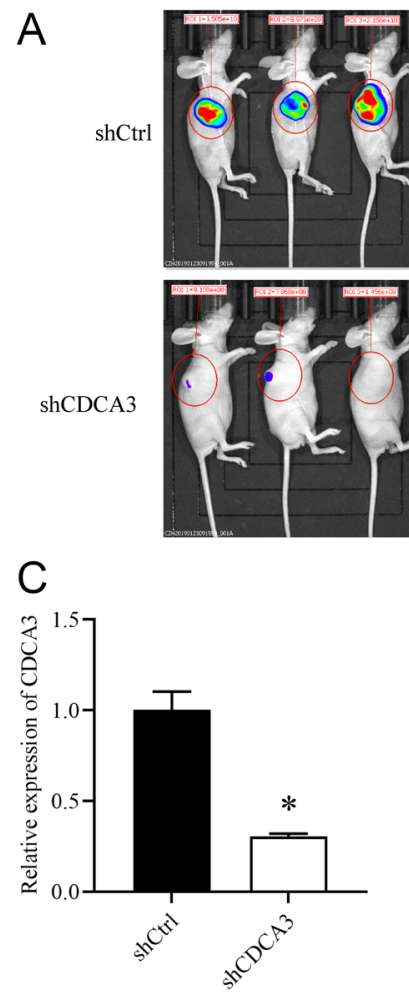

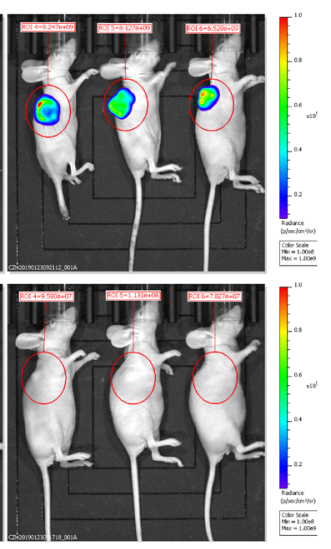

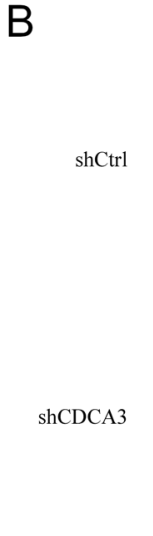

D

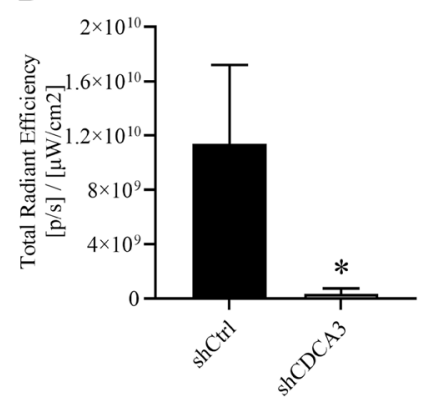

E

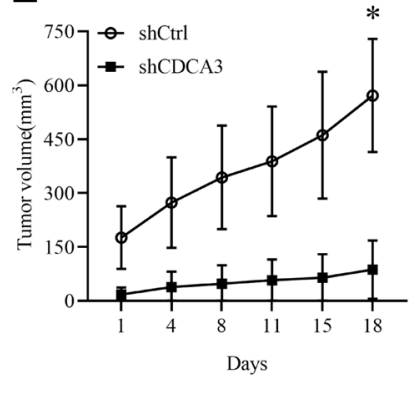

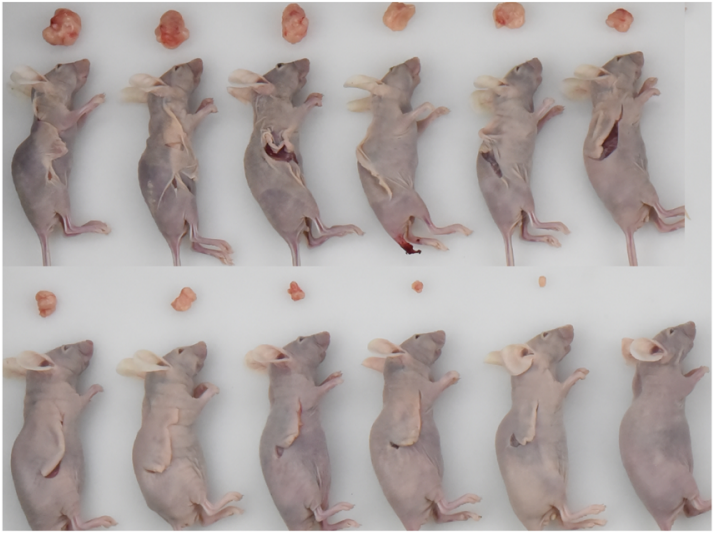

$\mathrm{F}$

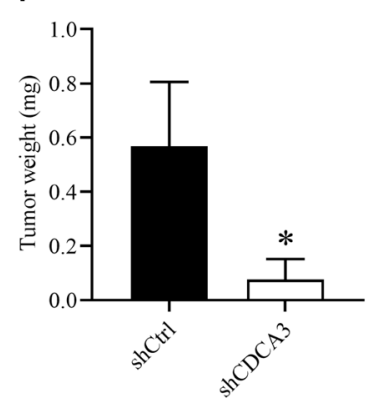

Figure 5. CDCA3 downregulation inhibited tumorigenesis in nude mice in vivo. A) The total radiation efficiency of xenograft tumor injected with shCDCA3 and shCtrl SW 1990 cells; B) Images of shCDCA3 and shCtrl groups nude mice; C) The mRNA expression of CDCA3 in SW 1990 before injecting; D) Quantitative data of fluorescence imaging analysis in xenograft tumors; E-F) The effect of CDCA3 downregulation on tumor volume and weight after subcutaneous transplantation. ${ }^{\star} \mathrm{p}<0.05$, compared with shCtrl group.

opment of PAC. Additionally, the RNA-Seq analysis results showed that multiple genes were significantly differentially expressed, and ingenuity pathway analysis suggested that these genes expression changed significantly in 12 signaling pathways. However, the precise mechanisms have not been fully illustrated. Therefore, to clarify the special function and regulatory networks related to CDCA 3 could help to provide new strategies for curing PAC.

CDCA3 is essential to regulate plenty of physiological and pathological processes by stimulating the protein degradation like transcription factors and signal transduction molecules [33], and exert an important role during various cancer types development $[19,22]$. Some researches indicated that CDCA3 was markedly overexpressed accompanied by poor prognosis in NSCLC, and exerted a vital part during tumorigenesis and esophageal carcinoma development [34]. Zhang et al. found that CDCA3 upregulation accelerated cell proliferation and colony formation, while CDCA3 knockdown reversed those effects [35]. Additionally, related studies showed the expression of CDCA3 was significantly increased in hepatocellular carcinoma [19] and prostate cancer [20]. In the present study, a series of biological experiments were performed, and the results revealed that CDCA3 was obviously upregulated in PAC tissues compared to peritu- moral tissue, and lower expression of CDCA3 was associated with longer survival time, and lower cell activity and tumor weight. The data strongly implied that CDCA3 may serve as an oncogene in PAC.

Furthermore, to address the underlying mechanism, the microarray and ingenuity pathway analysis were employed to detect the genes and pathway alteration elicited by the CDCA3 knockdown. The results showed that Xbp1 and KLF6 were closely associated with the CDCA3 knockdown in PAC. XBP1 plays an important part in tumorigenesis and development by regulating apoptotic genes and cell cycle [36, 37]. Studies in recent years demonstrated that the dysregulation of XBP1 was positively associated with the proliferation and tumor cells apoptosis, epithelial-mesenchymal transition (EMT), and regulation of transcription factors [38, 39]. Besides, KLF6 may serve as a tumor suppressor implicated with signal transduction, proliferation, differentiation, and cell death in various tumors $[40,41]$. Guo pointed out that OR3A4 enhanced the cell metastasis and invasion of ovarian cancer through inhibiting KLF6 expression [42]. And KLF6 suppressed clear cell renal cell carcinoma metastasis through suppressing E2F1 expression [43]. Therefore, we speculated that CDCA3 downregulation may contribute to the anomalous expression of XBP1 and KLF6, and thereby induce the 
disorder of PAC apoptosis and proliferation. Nevertheless, whether this view was correct, more researches are required and the intricate relation is still needed to be deeply illustrated. Collectively, our results provided a molecular link between these genes and gave clues to the potential molecular mechanism, and these findings eventually lay down a theoretic foundation for developing effective anticancer drugs about PAC.

In conclusion, in this study, we provide the first confirmation that the expression of CDCA3 in tumor tissues was higher than that of normal tissues. Our results suggest that CDCA3 plays a crucial role in the progression of PCA by regulating cell apoptosis and proliferation. Further exploration of the molecular mechanisms underlying the oncogene effects of CDCA3 on PCA could lead to the identification of novel therapeutic targets to prevent or treat PCA.

Acknowledgments: This work was supported by the National Natural Science Foundation of China (81860423), and the Joint Project of Science and Technology, Department of Yunnan and Kunming Medical University (2017FE467(-183)), and The fund of Yunnan Provincial Clinical Center of Hepato-biliary-pancreatic Diseases, and the Ph.D. Student Innovation Fund of Kunming Medical University, Grant number: 2019D004, and the Doctor Newcomer Award of Yunnan Province in 2017.

\section{References}

[1] GAO J, WANG G, WU J, ZUO Y, ZHANG J et al. Skp2 Expression Is Inhibited by Arsenic Trioxide through the Upregulation of miRNA-330-5p in Pancreatic Cancer Cells. Mol Ther Oncolytics 2019; 12: 214-223. https://doi.org/10.1016/j. omto.2019.01.006

[2] SIEGEL RL, MILLER KD, JEMAL A. Cancer Statistics, 2017. CA Cancer J Clin 2017; 67: 7-30. https://doi.org/10.3322/ caac. 21387

[3] BRAY F, FERLAY J, SOERJOMATARAM I, SIEGEL RL, TORRE LA et al. Global cancer statistics 2018: GLOBOCAN estimates of incidence and mortality worldwide for 36 cancers in 185 countries. CA Cancer J Clin 2018; 68: 394-424. https://doi.org/10.3322/caac.21492

[4] BRAY F, FERLAY J, SOERJOMATARAM I, SIEGEL RL, TORRE LA et al. Global cancer statistics 2018: GLOBOCAN estimates of incidence and mortality worldwide for 36 cancers in 185 countries. CA Cancer J Clin 2018; 68: 394-424. https://doi.org/10.3322/caac.21492

[5] SIEGEL RL, MILLER KD, JEMAL A. Cancer statistics, 2018. CA Cancer J Clin 2018; 68: 7-30. https://doi.org/10.3322/ caac. 21442

[6] LIN QJ, YANG F, JIN C, FU DL. Current status and progress of pancreatic cancer in China. World J Gastroenterol 2015; 21: 7988-8003. https://doi.org/10.3748/wjg.v21.i26.7988

[7] LI D, XIE K, WOLFF R, ABBRUZZESE JL. Pancreatic cancer. Lancet 2004; 363: 1049-1057. https://doi.org/10.1016/ S0140-6736(04)15841-8
[8] MCWILLIAMS RR, MAISONNEUVE P, BAMLET WR, PETERSEN GM, LI D et al. Risk Factors for Early-Onset and Very-Early-Onset Pancreatic Adenocarcinoma: A Pancreatic Cancer Case-Control Consortium (PanC4) Analysis. Pancreas 2016; 45: 311-316. https://doi.org/10.1097/ MPA.0000000000000392

[9] MOLNAR I, HEGYI G, ZSOM L, SAAHS C, VAGEDES J et al. Celiac plexus block increases quality of life in patients with pancreatic cancer. J Pain Res 2019; 12: 307-315. https:// doi.org/10.2147/JPR.S186659

[10] AHMED AE, ALZAHRANI FS, GHARAWI AM, ALAMMARY SA, ALMIJMAJ FH et al. Improving risk prediction for pancreatic cancer in symptomatic patients: a Saudi Arabian study. Cancer Manag Res 2018; 10: 4981-4986. https:// doi.org/10.2147/CMAR.S173666

[11] MACCHIA G, SAINATO A, TALAMINI R, BOZ G, BACIGALUPO A et al. Patterns of radiotherapy practice for pancreatic cancer: Results of the Gastrointestinal Radiation Oncology Study Group multi-institutional survey. Oncol Rep 2015; 34: 382-390. https://doi.org/10.3892/or.2015.3955

[12] RAWLAP,SUNKARAT,GADUPUTIV.EpidemiologyofPancreaticCancer:GlobalTrends, EtiologyandRiskFactors. World J Oncol 2019; 10: 10-27. https://doi.org/10.14740/wjon1166 [13] HIDALGO M, CASCINU S, KLEEFF J, LABIANCA R, LOHR JM et al. Addressing the challenges of pancreatic cancer: future directions for improving outcomes. Pancreatology 2015; 15: 8-18. https://doi.org/10.1016/j.pan.2014.10.001

[14] HUI B, XU Y, ZHAO B, JI H, MA Z et al. Overexpressed long noncoding RNA TUG1 affects the cell cycle, proliferation, and apoptosis of pancreatic cancer partly through suppressing RND3 and MT2A. Onco Targets Ther 2019; 12: 1043-1057. https://doi.org/10.2147/OTT.S188396.

[15] CAI J, LU W, DU S, GUO Z, WANG H et al. Tenascin-C Modulates Cell Cycle Progression to Enhance Tumour Cell Proliferation through AKT/FOXO1 Signalling in Pancreatic Cancer. J Cancer 2018; 9: 4449-4462. https://doi. org/10.7150/jca.25926

[16] AYAD NG, RANKIN S, MURAKAMI M, JEBANATHIRAJAH J, GYGI $S$ et al. Tome-1, a trigger of mitotic entry, is degraded during G1 via the APC. Cell 2003; 113: 101-113. https://doi.org/10.1016/s0092-8674(03)00232-0

[17] YOSHIDA K. Cell-cycle-dependent regulation of the human and mouse Tome-1 promoters. FEBS Lett 2005; 579: 14881492. https://doi.org/10.1016/j.febslet.2005.01.055

[18] LIM HH, SURANA U. Tome-1, wee1, and the onset of mitosis: coupled destruction for timely entry. Mol Cell 2003; 11 : 845-846. https://doi.org/10.1016/s1097-2765(03)00149-7

[19] HU Q, FU J, LUO B, HUANG M, GUO W et al. OY-TES-1 may regulate the malignant behavior of liver cancer via NANOG, CD9, CCND2 and CDCA3: a bioinformatic analysis combine with RNAi and oligonucleotide microarray. Oncol Rep 2015; 33: 1965-1975. https://doi.org/10.3892/ or. 2015.3792

[20] CHEN J, ZHU S, JIANG N, SHANG Z, QUAN C et al. HoxB3 promotes prostate cancer cell progression by transactivating CDCA3. Cancer Lett 2013; 330: 217-224. https:// doi.org/10.1016/j.canlet.2012.11.051 
[21] UCHIDA F, UZAWA K, KASAMATSU A, TAKATORI H, SAKAMOTO Y et al. Overexpression of cell cycle regulator CDCA3 promotes oral cancer progression by enhancing cell proliferation with prevention of G1 phase arrest. BMC Cancer 2012; 12: 321. https://doi.org/10.1186/1471-2407-12-321

[22] ADAMS MN, BURGESS JT, HE Y, GATELY K, SNELL C et al. Expression of CDCA3 Is a Prognostic Biomarker and Potential Therapeutic Target in Non-Small Cell Lung Cancer. J Thorac Oncol 2017; 12: 1071-1084. https://doi.org/10.1016/j. jtho.2017.04.018

[23] AZIM HJ, PECCATORI FA, BROHEE S, BRANSTETTER $\mathrm{D}$, LOI $\mathrm{S}$ et al. RANK-ligand (RANKL) expression in young breast cancer patients and during pregnancy. Breast Cancer Res 2015; 17: 24. https://doi.org/10.1186/s13058-015-0538-7

[24] RITCHIE ME, PHIPSON B, WU D, HU Y, LAW CW et al. limma powers differential expression analyses for RNAsequencing and microarray studies. Nucleic Acids Res 2015; 43: e47. https://doi.org/10.1093/nar/gkv007

[25] XU A, SUN S. Genomic profiling screens small molecules of metastatic prostate carcinoma. Oncol Lett 2015; 10: $1402-$ 1408. https://doi.org/10.3892/ol.2015.3472

[26] KRAMER A, GREEN J, POLLARD JJ, TUGENDREICH S. Causal analysis approaches in Ingenuity Pathway Analysis. Bioinformatics 2014; 30: 523-530. https://doi.org/10.1093/ bioinformatics/btt703

[27] FELCIANO RM, BAVARI S, RICHARDS DR, BILLAUD JN, WARREN T et al. Predictive systems biology approach to broad-spectrum, host-directed drug target discovery in infectious diseases. Pac Symp Biocomput 2013; 17-28.

[28] STEFANIUS K, SERVAGE K, DE SOUZA SM, GRAY HF, TOOMBS JE et al. Human pancreatic cancer cell exosomes, but not human normal cell exosomes, act as an initiator in cell transformation. Elife 2019; 8: e40226. https://doi. org/10.7554/eLife.40226

[29] WU W, HE X, YANG L, WANG Q, BIAN X et al. Rising trends in pancreatic cancer incidence and mortality in 2000-2014. Clin Epidemiol 2018; 10: 789-797. https://doi. org/10.2147/CLEP.S160018

[30] JIN HB, LU L, XIE L, YANG JF, ZHANG XF et al. Concentration changes in gemcitabine and its metabolites after hyperthermia in pancreatic cancer cells assessed using RP-HPLC. Cell Mol Biol Lett 2019; 24: 30. https://doi. org/10.1186/s11658-019-0153-1

[31] KLEIN AP, BRUNE KA, PETERSEN GM, GOGGINS M, TERSMETTE AC et al. Prospective risk of pancreatic cancer in familial pancreatic cancer kindreds. Cancer Res 2004; 64: 2634-2638. https://doi.org/10.1158/0008-5472.can-03-3823

[32] VINCENT A, HERMAN J, SCHULICK R, HRUBAN RH, GOGGINS M. Pancreatic cancer. Lancet 2011; 378: 607-620. https://doi.org/10.1016/S0140-6736(10)62307-0
[33] ZHENG N, SCHULMAN BA, SONG L, MILLER JJ, JEFFREY PD et al. Structure of the Cul1-Rbx1-Skp1-F boxSkp2 SCF ubiquitin ligase complex. Nature 2002; 416: 703-709. https://doi.org/10.1038/416703a

[34] CHEN X, CAI S, LI B, ZHANG X, LI W et al. Identification of key genes and pathways for esophageal squamous cell carcinoma by bioinformatics analysis. Exp Ther Med 2018; 16: 1121-1130. https://doi.org/10.3892/etm.2018.6316

[35] ZHANG Y, YIN W, CAO W, CHEN P, BIAN L et al. CDCA3 is a potential prognostic marker that promotes cell proliferation in gastric cancer. Oncol Rep 2019; 41: 2471-2481. https://doi.org/10.3892/or.2019.7008

[36] SHAJAHAN AN, RIGGINS RB, CLARKE R. The role of $\mathrm{X}$-box binding protein-1 in tumorigenicity. Drug News Perspect 2009; 22: 241-246. https://doi.org/10.1358/ dnp.2009.22.5.1378631

[37] KURATA M, YAMAZAKI Y, KANNO Y, ISHIBASHI S, TAKAHARA T et al. Anti-apoptotic function of Xbp1 as an IL-3 signaling molecule in hematopoietic cells. Cell Death Dis 2011; 2: e118. https://doi.org/10.1038/cddis.2011.1

[38] CHEN X, ILIOPOULOS D, ZHANG Q, TANG Q, GREENBLATT MB et al. XBP1 promotes triple-negative breast cancer by controlling the HIF1alpha pathway. Nature 2014; 508: 103-107. https://doi.org/10.1038/nature13119

[39] MARUMOTO Y, TERAI S, URATA Y, MATSUMOTO T, MIZUNAGA $Y$ et al. Continuous high expression of XBP1 and GRP78 is important for the survival of bone marrow cells in CCl4-treated cirrhotic liver. Biochem Biophys Res Commun 2008; 367: 546-552. https://doi.org/10.1016/j. bbrc.2007.12.171

[40] KIMMELMAN AC, QIAO RF, NARLA G, BANNO A, LAU $\mathrm{N}$ et al. Suppression of glioblastoma tumorigenicity by the Kruppel-like transcription factor KLF6. Oncogene 2004; 23 : 5077-5083. https://doi.org/10.1038/sj.onc. 1207662

[41] KREMER-TAL S, REEVES HL, NARLA G, THUNG SN, SCHWARTZ $M$ et al. Frequent inactivation of the tumor suppressor Kruppel-like factor 6 (KLF6) in hepatocellular carcinoma. Hepatology 2004; 40: 1047-1052. https://doi. org/10.1002/hep.20460

[42] GUO FF, JIANG MM, HONG LL, QIAO B, LIN XM et al. Long non-coding RNA OR3A4 promotes metastasis of ovarian cancer via inhibiting KLF6. Eur Rev Med Pharmacol Sci 2019; 23: 2360-2365. https://doi.org/10.26355/eurrev_201903_17380

[43] GAO Y, LI H, MA X, FAN Y, NI D et al. KLF6 Suppresses Metastasis of Clear Cell Renal Cell Carcinoma via Transcriptional Repression of E2F1. Cancer Res 2017; 77: 330-342. https://doi.org/10.1158/0008-5472.CAN-16-0348 